\title{
Generalized Axisymmetric Spacetimes *
}

\author{
Franco Bampi and Roberto Cianci \\ Istituto Matematico dell 'Università I-16132 Genova, Italy
}

\begin{abstract}
Properties of space-times admitting a two parameter abelian symmetry group acting on a null two-surface are investigated. Within this framework, a class of new solutions of Einstein's vacuum equations are found. Some analogies with the theory of black hole are pointed out.
\end{abstract}

\section{Introduction}

One of the most useful techniques for finding new solutions of Einstein's gravitational equations is surely to require that the space-time admits an assigned group of symmetry, namely a suitable set of Killing vector fields. This kind of procedure has given excellent results even if the space-time admits a group of symmetry depending on two parameters only. The Lewis line element [1]

$$
d s^{2}=e^{\mu}\left(d x^{1}\right)^{2}+e^{v}\left(d x^{2}\right)^{2}+\tilde{X} d \phi^{2}+2 \widetilde{W} d \phi d t-\widetilde{V} d t^{2}
$$

where $\tilde{V}, \tilde{W}, \tilde{X}, \mu, v$ are functions of $x^{1}, x^{2}$ only, is undoubtedly the most celebrated example: indeed, under very weak and general assumptions, the final state of a stationary rotating black hole can be exhibited in the previous form (see prop. 9.3.6. of [2]).

The line element $(1.1)$ is uniquely determined by the following properties:

$\left(a_{1}\right)$ there exists a two parameter abelian symmetry group;

$\left(a_{2}\right)$ the generators of the group $k^{i}$ and $m^{i}$ satisfy the circularity condition

$$
\begin{aligned}
& k_{[a ; b k c m d]}=0, \\
& m_{[a ; b k c m d]}=0 .
\end{aligned}
$$

$\left(a_{3}\right)$ the two-surfaces of transitivity $V_{2}$ of the group are non-null.

From a geometrical viewpoint, the previous properties are the conditions that there exists a family of two-surfaces everywhere orthogonal to the surfaces of transitivity of the group (orthogonal transitivity condition [3]). Notice that orthogonal transitivity does not hold when the surface $V_{2}$ becomes null: this is

\footnotetext{
* This work was carried out under the auspices of the National Group of Mathematical Physics
} of C.N.R. 
tantamount to require that $\tilde{V} \tilde{X}+\tilde{W}^{2}=0$, which, in turn, makes Lewis' line element singular. This is the actual behaviour of the metric (1.1) on the horizon of an axisymmetric black hole.

In this paper we analyse the class of metrics satisfying $\left(a_{1}\right),\left(a_{2}\right)$ and the following:

$\left(a_{3}\right)^{\prime}$ the two-surfaces of transitivity $V_{2}^{*}$ of the group are null.

The most general line element which satisfies conditions $\left(a_{1}\right),\left(a_{2}\right),\left(a_{3}\right)^{\prime}$ may be found in Petrov's book [4]. By straightforward transformations, the metrics (29.2) and (29.4) of [4] can be exhibited in the form

$$
d s^{2}=2(S(r, z) d u+P(r, z) d v+X(r, z) d r) d r+V^{2}(r, z)(R(r) d u+d v)^{2}+d z^{2} / q^{2}(z)
$$

$\partial / \partial u, \partial / \partial v$ being the Killing vector fields. Notice that, in the case $R=0$, the field $\partial / \partial u$ is null and $\partial / \partial v$ is spacelike; on the contrary, when $R \neq 0$, the fields $\partial / \partial u, \partial / \partial v$ are both spacelike, besides no generality is lost by choosing $R(r)=r$.

Section 2 is devoted to solving Einstein's vacuum equations assuming the line element in the form (1.3). The solutions of type II, all of which have complex dilatation $\rho=0$, are believed to be new. It is worth remarking that the solutions which can be obtained from the Ernst theory $[5,6]\left(\tilde{V} \tilde{X}+\widetilde{W}^{2}=\right.$ variable), the Hoffman solutions $[7]\left(\tilde{V} \tilde{X}+\widetilde{W}^{2}=\right.$ const. $\left.\neq 0\right)$, and the solutions found here $\left(\tilde{V} \tilde{X}+\tilde{W}^{2}=0\right)^{1}$ exhaust the class of metrics satisfying $\left(a_{1}\right),\left(a_{2}\right)$. In section 3 we investigate the possibility of interpreting the metric (1.3) as a space-time filled by "horizons" with zero surface gravity. Finally, for the convenience of the reader, we give, in Appendix, the expression for the curvature two-forms and for the Newman-Penrose components of the vacuum Riemann tensor.

\section{Vacuum Metrics}

In this section we solve Einstein's vacuum equations for the metric (1.3). The differential system (A.7) $\div$ (A.12), we are dealing with, is listed in Appendix.

Case $R=0$.

Choosing $q=1$, equation (A.8) is trivially satisfied if $S_{z}=0$. This assumption immediately leads to type $N$ metrices with $\rho=\tau=0$ (i.e. $p p$-waves). They were studied exhaustively by Kundt in [9] (see also [10]).

Let us consider the case $S_{z} \neq 0$. Equations (A.8), (A.9), (A.10) may be solved straightforwardly. Comparison with (A.11) yields:

$$
\begin{aligned}
& V=\beta(z+\delta(r))^{-1 / 3} \\
& S=\varepsilon(r)(z+\delta(r))^{4 / 3}
\end{aligned}
$$

where $\beta$ is an arbitrary constant and $\delta(r), \varepsilon(r)$ are arbitrary functions. Now, equation (A.7) gives:

$$
P=(z+\delta)^{4 / 3}\left[\lambda(r)+\mu(r)(z+\delta)^{-2}\right]
$$

1 Although the form (1.1) is not available for the metric at hand, the obvious definitions [8] $\tilde{V}=-k_{i} k^{i}$, $\tilde{X}=m_{i} m^{i} \tilde{W}=k_{i} m^{i}$ are retained also in the present case. 
$\lambda(r), \mu(r)$ being arbitrary functions. Finally, by means of (A.12), we have:

$$
\begin{aligned}
X= & \alpha(r)(z+\delta)^{4 / 3}-\gamma(r)(z+\delta)^{4 / 3} \ln |z+\delta|+3(z+\delta)\left(\delta_{r r}-\delta_{r} \varepsilon_{r} / \varepsilon\right) \\
& +\left(\mu^{2} / 2\right)(z+\delta)^{-2 / 3}-\delta_{r}^{2} / 2 .
\end{aligned}
$$

Once more $\alpha(r), \gamma(r)$ are arbitrary functions. In general, the resulting metrics are of type II with $\rho=0$; particular choice of the arbitrary functions specialize the metric to type $D$ only.

Case $R=r$.

In the present case we limit our analysis to the choice $P=0$ in the line element $(1.3)^{2}$. Using equations (A.7), (A.8), (A.10) we obtain:

$$
\begin{aligned}
& V^{3}=p^{3}(r) q(z) S_{z} / S \\
& S=p^{3}(r) /[w(z)+m(r)]
\end{aligned}
$$

where $w(z), m(r), p(r)$ are arbitrary functions. Now, inserting (2.5), (2.6) in (A.8), a straightforward but tedious calculation shows that $m$ and $p$ are constant. This implies that $V$ and $S$ are functions of $z$ only: hence equations (A.7), (A.11) are trivially satisfied.

It is worth noticing that an attempt to solve the differential system (A.8), (A.9), (A.10), choosing quite naturally $q=1$, gives the quantity $S_{z} / S$ in terms of elliptic integrals. To avoid this difficulty, we choose $S$ to be the coordinate $z$. In this way, sostitution of (2.5) in (A.8) gives a first order equation for $q(z)$ only: the integration is now straightforward. Explicitly we obtain:

$$
q=z^{-1 / 2}\left(c z-p^{2}\right)^{3 / 4},
$$

$c$ being an arbitrary constant. In view of this result, equation (A.9) is identically satisfied and equation (2.5) yields:

$$
V=p z^{-1 / 2}\left(c z-p^{2}\right)^{1 / 4} \text {. }
$$

Finally, due to the previous results, equation (A.12) yields:

$$
X=z\left[\alpha(r)+\varepsilon(r) \ln \left|c z-p^{2}\right|\right],
$$

$\alpha(r), \varepsilon(r)$ being arbitrary functions. Actually $\alpha(r)$ is inessential. In fact, by performing the coordinate transformation

$$
\begin{aligned}
& u \rightarrow u-\int \alpha(r) d r, \\
& v \rightarrow v+\int \alpha(r) r d r, \\
& r \rightarrow r,
\end{aligned}
$$

the line element corresponding to the ansatz $\alpha \neq 0$ is reduced to the one which can be obtained by putting $\alpha=0$. We point out that the choice $\varepsilon(r)=0$ leads to a type D metric, namely the metric IV A of Kinnersley's catalogue [11]. The general case $\varepsilon \neq 0$ is a type II metric with $\rho=0$.

2 The general case $P \neq 0$ is very cumbersome, hardly practicable and will be not considered here. 


\section{Comments}

The structure of the metric (1.3) allows us to derive the following results:

$\left(b_{1}\right)$ for every $V_{2}^{*}$ there exists a null three-surface $H$, given by $r=$ const., such that $V_{2}^{*} \subset H$;

$\left(b_{2}\right)$ the vector field normal to $H$ is $\ell=\partial / \partial u-R(r) \partial / \partial v$;

$\left(b_{3}\right) \ell$ is geodesics, non-diverging $(\rho=0)$, and affine parametrized.

In view of the evident analogy with the properties of a stationary black hole $[2,8]$, one could be led to determine whether the surfaces $H$ represent horizons. If this were the case, adopting for every $H, r=r_{0}$, the well known definitions of temperature $\kappa$ (surface gravity) and angular velocity $\Omega$, one could show that $\kappa=0$ and $\Omega=R\left(r_{0}\right)$. However this interpretation does not seem possible in the vacuum case. Indeed, if $H$ were an horizon, its topology should be $S^{2} \times \mathbb{R}$. Consider now the slice $\Sigma$ given by $r=$ const. and $u=$ const. : as $\Sigma$ is spacelike we should require its topology to be $S^{2}$ [12]. Unfortunately, independently of topological identifications on the coordinate $v$, the two-area of $\Sigma$ is always unbounded. In the case $R=0$ the proof is straightforward. On the other hand, even in the general case $R=r, P \neq 0$, it is possible to show that, setting $q=1, \zeta=S-r P$, the metric on $\Sigma$ becomes:

$$
d \sigma^{2}=p^{2}(r) \zeta^{-1}\left(c(r) \zeta-p^{2}(r)\right)^{1 / 2} d v^{2}+\zeta\left(c(r) \zeta-p^{2}(r)\right)^{-3 / 2} d \zeta^{2}
$$

where $c(r), p(r)$ are arbitrary functions: then the proof is again straightforward.

On purely physical ground, it is our opinion that the right topology on $H$ could be obtained only by using some pathological energy momentum tensors [13].

\section{Appendix}

We consider the following metric:

$$
d s^{2}=2 \omega^{0} \omega^{1}+\left(\omega^{2}\right)^{2}+\left(\omega^{3}\right)^{2}
$$

where

$$
\begin{aligned}
& \omega^{0}=d r \\
& \omega^{1}=S(r, z) d u+P(r, z) d v+X(r, z) d r, \\
& \omega^{2}=V(r, z)(R(r) d u+d v), \\
& \omega^{3}=d z / q(z) .
\end{aligned}
$$

By means of Cartan's calculus [14], we obtain the following connection oneforms:

$$
\begin{array}{ll}
\omega_{0}^{0}=a \omega^{0}+f / 2 \omega^{2}-d / 2 \omega^{3}, & \omega^{1}{ }_{2}=-b \omega^{0}-f / 2 \omega^{1}-g \omega^{2}-e / 2 \omega^{3} \\
\omega^{0}{ }_{2}=f / 2 \omega^{0} & \omega^{1}{ }_{3}=-c \omega^{0}-d / 2 \omega^{1}-e / 2 \omega^{2} \\
\omega^{0}{ }_{3}=-d / 2 \omega^{0} & \omega^{2}{ }_{3}=-e / 2 \omega^{0}-h \omega^{2}
\end{array}
$$


where

$$
\begin{array}{rlrl}
a & =\frac{S_{r}-R P_{r}}{S-R P} & e & =q \frac{P S_{z}-S P_{z}}{V(S-R P)} \\
b & =\frac{S P_{r}-P S_{r}}{V(S-R P)} & f & =\frac{V R_{r}}{S-R P} \\
c & =q X \frac{S_{z}-R P_{z}}{S-R P}-q X_{z} & g & =\frac{V_{r}}{V}-\frac{P R_{r}}{S-R P} \\
d & =-q \frac{S_{z}-R P_{z}}{S-R P} & h & =-q \frac{V_{z}}{V} .
\end{array}
$$

The curvature two-forms follows at once, namely:

$$
\begin{aligned}
& \theta_{0}^{0}=A \omega^{0} \wedge \omega^{1}+B \omega^{0} \wedge \omega^{2}+C \omega^{0} \wedge \omega^{3}+2 D \omega^{2} \wedge \omega^{3} \\
& \theta_{2}^{0}=E \omega^{0} \wedge \omega^{2}+D \omega^{0} \wedge \omega^{3} \\
& \theta_{3}^{0}=-D \omega^{0} \wedge \omega^{2}+F \omega^{0} \wedge \omega^{3} \\
& \theta^{1}{ }_{2}=-B \omega^{0} \wedge \omega^{1}+G \omega^{0} \wedge \omega^{2}+H \omega^{0} \wedge \omega^{3}+E \omega^{1} \wedge \omega^{2}-D \omega^{1} \wedge \omega^{3} \\
& +J \omega^{2} \wedge \omega^{3} \\
& \theta^{1}{ }_{3}=-C \omega^{0} \wedge \omega^{1}+H \omega^{0} \wedge \omega^{2}+K \omega^{0} \wedge \omega^{3}+D \omega^{1} \wedge \omega^{2}+F \omega^{1} \wedge \omega^{3} \\
& +L \omega^{2} \wedge \omega^{3} \\
& \theta_{3}^{2}=-2 D \omega^{0} \wedge \omega^{1}+J \omega^{0} \wedge \omega^{2}+L \omega^{0} \wedge \omega^{3}+M \omega^{2} \wedge \omega^{3} \text {, } \\
& A=3 f^{2} / 4-d^{2} / 4 \quad G=-g_{r}+a g-b f-g^{2}-c h+e^{2} / 4 \\
& B=f_{r} / 2+f g-d e / 4 \quad H=q b_{z}+e_{r} / 2-c f / 2-a e / 2+b d / 2 \\
& C=d_{r} / 2+3 e f / 4 \quad J=q g_{z}-3 e f / 4-g h+d g / 2 \\
& D=-q f_{z} / 4+f h / 4 \quad K=q c_{z}+e^{2} / 4 \\
& E=-f^{2} / 4-d h / 2 \quad L=q e_{z} / 2-d e / 2 \\
& F=q d_{z} / 2-d^{2} / 4 \quad M=q h_{z}-h^{2}
\end{aligned}
$$

Einstein vacuum equations may be cast in the following form:

$$
\begin{aligned}
& B+L=0 \\
& A+2 E=0 \\
& A-M=0 \\
& E-F=0 \\
& C-J=0 \\
& G+K=0
\end{aligned}
$$

Introducing the null tetrad $\omega^{0},-\omega^{1}, \omega^{2} \pm i \omega^{3}$, in the vacuum case the Newman 
-Penrose components of the Weyl tensor read [15]:

$$
\begin{aligned}
& \Psi_{0}=0, \quad \Psi_{1}=0, \quad \Psi_{2}=\frac{1}{2} A+i D \\
& \Psi_{3}=2^{-1 / 2}(B-i C), \quad \Psi_{4}=-G+i H
\end{aligned}
$$

Remark. For the sake of completeness, we analyze space-times which satisfy $\left(a_{1}\right)$ and $\left(a_{3}\right)^{\prime}$ but not $\left(a_{2}\right)$. In this case the line element is [4]:

$$
d s^{2}=2 \omega^{0} \omega^{1}+\left(\omega^{2}\right)^{2}+\left(\omega^{3}\right)^{2},
$$

where $\omega^{0}=d z, \omega^{1}=\tilde{A} d u+\widetilde{B} d r, \omega^{2}=\tilde{C}(r d u+d v)+\widetilde{D} d r, \omega^{3}=\widetilde{E}(r d u+d v) ; \tilde{A}, \tilde{B}$, $\tilde{C}, \tilde{D}, \widehat{E}$ being functions of $r, z$ only. Now, a straightforward calculation of the anholonomic component $G_{11}=R_{11}=R_{1 p 1}^{p}$ yields:

$$
G_{11}=-\frac{1}{2}\left(\widetilde{E}^{2}+\widetilde{C}^{2}\right)^{2}(\tilde{A} \tilde{D} \tilde{E})^{-2}
$$

which shows at once that there exist no vacuum metrics. Moreover, taking into account Einstein's equations $G_{i j}=8 \pi T_{i j}$, it is an easy matter to verify that the weak energy condition $\left(T_{i j} k^{i} k^{j} \geqq 0, k\right.$ non-spacelike [2]) is never satisfied assuming $k=g^{-1}\left(\omega^{0}\right)=\tilde{A}^{-1}(\partial / \partial u-r \partial / \partial v)$.

\section{References}

1. Lewis, T. : Proc. Roy. Soc. (London), A 136, 176-192 (1932)

2. Hawking, S. W. \& Ellis G. F. R. : The large scale structure of space-time. Cambridge University Press 1973

3. Carter, B. : J. Math. Phys. 10, 70-81 (1969)

4. Petrov, A. Z. : Einstein spaces. Oxford : Pergamon Press 1969

5. Ernst, F. J. : Phys. Rev. 167, 1175-1178 (1968); Phys. Rev. 168, 1415-1417 (1968)

6. Reina, C., Treves A. : Gen. Rel. Grav. 7, 817-838 (1976)

7. Hoffman, R. B. : J. Math. Phys. 10, 953-956 (1969)

8. Carter, B.: Black hole equilibrium states. In: Black holes. (eds. C. M. De Witt,. B. S. De Witt). New York : Gordon \& Breach 1973

9. Kundt W. : Z. Physik 163, 77-86 (1961)

10. Ehlers J., Kundt W. : Exact solutions of the gravitational field equations. In : Gravitation : an introduction to the current research. (ed. L. Witten). New York : John Wiley 1962

11. Kinnersley W. : J. Math. Phys. 10, 1195-1203 (1969)

12. Hawking S. W. : The event horizon. In : Black holes. (eds. C. M. De Witt, B. S. De Witt). New York : Gordon \& Breach 1973

13. Cianci R. : Riv. Matem. Univ. Parma (in print). See also Lauten W. T., Ray J. R. : J. Math. Phys. 18, 885-888 (1977)

14. Misner, C. W. : J. Math. Phys. 4, 924-937 (1963)

15. Newman, E. T., Penrose R. : J. Math. Phys. 3, 566-578 (1962)

Communicated by R. Geroch

Received April 17, 1979 